\title{
Diagnosis of hepatic glycogen storage disease patients with overlapping clinical symptoms by massively parallel sequencing: a systematic review of literature
}

\author{
Zahra Beyzaei $^{1} \mathbb{D}$, Bita Geramizadeh ${ }^{1,2^{*}}$ and Sara Karimzadeh ${ }^{3}$
}

\begin{abstract}
Background: Glycogen storage diseases (GSDs) with liver involvement are complex disorders with similar manifestations. Currently, the main diagnostic methods such as tissue diagnosis, either histopathology or enzyme assay, are invasive. Meanwhile, GSDs are diseases with significant genetic heterogeneity, and gene-sequencing methods can be more useful. This systematic review aims to review the literature to assess the value of massively parallel sequencing in the diagnosis of GSDs on patients with previously undiagnosed hepatic involvement.

Methods: Relevant studies identified in the MEDLINE/PubMed, EMBASE, Cochrane Library, Scopus, and Web of Science Core Collection databases up to July 2019 with no time and language restrictions. Publications were included in the review if they analyzed GSDs with hepatic involvement (GSD I, GSD III, GSD IV, GSD VI, GSD IX), using targeted gene sequencing (TGS) or exome sequencing (ES).

Results: Eleven studies were included in this systematic review. ES demonstrated a 93\% diagnostic yield. These methods correctly distinguished all types of pathogenic variants. The diagnostic yield of the TGS method was around $79.7 \%$.

Conclusions: According to our results, TGS analysis can be considered as the first-line diagnostic method with valuable results and ES can be used to diagnose complex cases of GSD with liver involvement. Overall, these molecular methods are considered as accurate diagnostic tools, which expedite correct diagnosis and treatment with significant cost-effectiveness by reducing unnecessary and inaccurate tests.
\end{abstract}

PROSPERO registration: CRD42020139931. Registered 8 January 2020.

Keywords: Glycogen storage disease (GSD), Massively parallel sequencing, Exome sequencing, Targeted gene sequencing, Rare disease diagnosis, Genetic diagnosis

\section{Background}

Glycogen storage disorders (GSDs) are a group of rare metabolic diseases with abnormal glycogen metabolism. The incidence of GSD is approximately 1:10,000 live

\footnotetext{
*Correspondence: geramib@gmail.com

${ }^{1}$ Transplant Research Center, Shiraz University of Medical Sciences, Shiraz, Iran

Full list of author information is available at the end of the article
}

births. These groups of diseases are caused by various enzyme deficiencies resulting in abnormal glycogen synthesis, or glycolysis, typically within the muscles and/or liver cells $[1,2]$. Different types of GSDs are categorized based on the type of deficient enzymes and affected tissues [3].

GSDs with liver involvement (Hepatic GSDs) are a complex group of disorders, including GSD Ia (G6PC, MIM \# 232200), Ib (SLC37A4, MIM \# 232220), III 
(AGL, MIM \# 232400), IV (GBE1, MIM \# 232500), VI (PYGL, MIM \# 232700), IXa (PHKA2, MIM \# 306000), IXb (PHKB, MIM \# 261750), and IXc (PHKG2, MIM \# 613027). All of them are associated with hypoglycemia and hepatomegaly [2]. Clinical signs of different types of hepatic GSDs are very similar, such as short fasting intervals (less than $4 \mathrm{~h}$ ), hepatomegaly or hypoglycemia, which can be observed in GSD type I as well as GSD III, IV, VI and IX. However, treatment methods and modalities, complications, and natural histories are different in various types of GSDs, which prompts definite and differential diagnosis between various types of GSDs. This can help to improve the quality of life, by decreasing the end-organ damage $[3,4]$. Moreover, these diseases create significant expenses for healthcare systems, which also prompts the necessity of precise diagnostic methods [5].

Currently, for accurate diagnosis of hepatic GSDs, a liver biopsy must be performed. Although the role of liver biopsy in the diagnosis of GSD is less common, however many publications still consider liver biopsy as the gold standard. Enzyme assay in the liver tissue is another option that can be measured for definite and decision-making diagnosis. Although the enzymatic activity of glucose-6-phosphatase (G6PC) for GSD Ia can be performed on frozen liver tissue, measuring glucose6-phosphate translocase (G6PT1) activity for GSD Ib is difficult to be performed on frozen liver samples and needs fresh liver tissue. Both of these necessitate liver biopsy, which is an invasive procedure, so the majority of clinical diagnostic laboratories do not assay the latter enzyme activity [2]. In addition, measurement of G6PC and G6PT1 as well as glycogen phosphorylase (PYGL) activity in the tissue samples is very laborious and requires a sophisticated laboratory. Molecular tests have rarely been used as the first diagnostic method or the method of choice in previous study reports $[4,6]$.

All hepatic GSDs, except for GSD IXa, are autosomal recessive. GSD IXa is an X-linked recessive disorder, so, molecular methods may provide a suitable procedure for the diagnosis and classification of hepatic GSDs. For many years, the conventional Sanger sequencing method has been the gold standard for the detection and screening of mutations. However, this method can only evaluate the exon-by-exon of one gene at a time, and some exons require multiple Sanger steps. Therefore, its validation for a mutational screening of large genes such as $A G L$ with 34 exons; the corresponding controls and necessary bidirectional reads would be very laborious [7]. In addition, due to the genetic heterogeneity of populations, consecutive tests of every candidate gene are costly and time-consuming, leading to a delayed diagnosis that decelerates care and treatment [2].
Compared with other molecular methods, massively parallel sequencing (MPS), also known as nextgeneration sequencing has the ability to simultaneously screen of large numbers of genes. It also adds unique gene sequence tags to each sample, allowing pooled testing and preventing invasive liver biopsies [2-8]. This pooling lets different patients to be sequenced together with simultaneous detection of other genomic alterations, e.g. screening GSD-associated genes and similar non-GSDassociated genes in one panel [7, 8]. Since 2009, MPS has been used for exome sequencing (ES), allowing targeted gene panels (TGS) to be sequenced faster in higher depth, which increases the sensitivity [9-11]. Despite all recent advances in MPS, the cost of sequencing is still remarkable and is different for various types of MPS technology [12]. Moreover, over time, there has been a significant decrease in sequencing costs, which has made the clinical application of MPS more practicable [1315]. Another important issue that must be considered is the depth of sequence coverage [16]. It is a fact that higher coverage of sequencing increases the validation of findings as well as costs. Therefore, investigators try to design clinical experiments with the best accuracy, coverage, and cost.

The whole evidence in the MPS application in patients with hepatic GSD has not been previously reviewed systematically. Therefore, it can be worthwhile to provide the best and most reliable objective analysis of the existing evidence from previous reports. For this purpose, we systematically reviewed the existing literature to assess the value of MPS in the diagnosis of GSDs in patients with previously undiagnosed hepatic involvement $[2,7,17-25]$. The review focused specifically on GSDs with hepatic involvement (GSD I, GSD III, GSD IV, GSD VI, GSD IX) diagnosed with exome sequencing (ES) or targeted gene sequencing (TGS).

\section{Materials and methods}

We conducted this study, according to the Preferred Reporting Items for Systematic Reviews and MetaAnalyses (PRISMA) guidelines [26] (Additional file 1). A complete protocol was registered at PROSPERO under the number CRD42020139931.

MPS is described to include ES and TGS. The ES panel consisted of all known associated disease genes available in the OMIM database until 2013. The TGS panel included all known genes in metabolic disorders, including GSD-associated genes, or only GSD-associated gene-disease panels with/or without genes related to its pathologic phenotypes panels. Different studies reported MPS only for probands or probands alongside their parents, siblings, or grandparents (duo or trio). This 
study is mainly based on the utilization of MPS for the diagnosis of GSDs with hepatic involvement.

\section{Search strategies and data sources}

We conducted an independent review of MEDLINE/ PubMed, EMBASE, Cochrane Library, Scopus, and Web of Science Core Collection databases with no time and language restrictions on November 30, 2018 and updated on July 31, 2019. The bibliography of the selected articles on the topic was manually searched for additional studies and for minimizing publication bias. The search strategy was designed and implemented by an experienced medical librarian using controlled keywords and the MeSH terms (Medical Subject Heading) from the Library of Shiraz Medical Center. One word (keyword) was identified by examining relevant references in the literature and the Medical Subject Headings (MeSH) used by EMBASE and MEDLINE (https://www.nlm. nih.gov/mesh/). The details of the search strategy are reported in Additional file 1.

\section{Inclusion and exclusion criteria}

Studies were included if they met the following criteria:

1 Peer-reviewed original research articles related to hepatic glycogen storage disease, including type I, III, IV, VI, IX, which has been diagnosed by MPS.

2 Case series related to hepatic glycogen storage disease, including type I, III, IV, VI, IX, which has been diagnosed by MPS.

3 Description/evaluation of the clinical application of MPS for diagnostic purposes with a proband. We also included articles related to carrier testing for hepatic GSDs, prenatal genetic testing, and targeted gene sequencing (e.g., "clinical exome" or "Mendeliome"), i.e. panels of thousands of genes known to be associated with single-gene disorders, provided they have included genes related to hepatic GSDs. There were no restrictions in selecting papers relative to their study design, including interventional studies (any methodology), and clinical reports (case series). Studies were excluded if they met the following criteria:

1 All studies reporting the use of MPS in the diagnosis of other types of GSDs, such as muscular forms (type III, IV) (i.e. not including hepatic GSDs).

2 All publications which have used only mitochondrial genome sequencing (i.e. without sequencing of the nuclear genome).
3 All the animal experiments, editorial pieces, commentaries, review articles, and symposium reports.

4 All case reports because these studies cannot determine the sensitivity and specificity of the tests.

Conference abstracts were included and evaluated in the protocol although none of them was eventually eligible. The literature search was undertaken in November 2018 and all citations were imported into EndNote (Clarivate Analytics, Boston, MA). Following deduplication, publications were scanned for relevance by title and abstract. Clearly, irrelevant publications were excluded. The full text of the remaining publications was then evaluated for relevance by both authors (ZB and BG) independently. The selected studies were comprehensively surveyed by both reviewers, and those, which fulfilled the eligibility criteria were selected for detailed data mining and the quality assessment. Disagreements at both stages were resolved by consensus and referring back to the original article.

\section{Methodological quality assessment}

Both authors (ZB and BG) independently conducted a quality assessment of the studies. For quality assessment, checklists were used which have been developed by the National Heart, Lung, and Blood Institute (NHLBI) Quality Assessment Tool for Observational Cohort and Cross-Sectional Studies. Cochrane (NHLBI 2014) has recommended these checklists [27]. The tools assessed each quality criterion as "Yes," "No," or "cannot be determined" "Not reported" or "Not applicable". Reviewers categorized them as: $>8$ yes $=$ Good, $7-8$ yesFair, and $<6$ yes $=$ Poor. The total agreement between the reviewers was $82 \%$ as shown in Additional file 1 . Meanwhile, as all the included articles were observational studies, the context and population structure were also considered.

\section{Data extraction}

According to the PRISMA guidelines, data extraction was independently carried out by the two authors (ZB, BG), using a data extraction form. Disagreements were resolved by consensus; if not, the original article was evaluated once more, and finally, the issue was resolved through discussion to reach the consensus. The collected data included bibliographic details, information about the first author name/year of publication, type of study/ GSD, number of patients, presentation of disease, country, mean age of the patients at molecular diagnostic test, consanguinity, measurement of enzyme activity, liver biopsy type of MPS/panel, sequencing methods, 
sequencing platforms, control database, MPS instrument brand, whether a duo and/or trio approach was used. The authors contacted the corresponding author of selected articles to get access to more details, if needed. The analysis was performed with Stata IC 15 (College Station, TX).

\section{Results}

\section{Study selection}

The study selection process is presented in Fig. 1 as a PRISMA flow diagram. In primary search, 1692 articles were identified, of which 431 articles were duplicated. After initial screening of the titles and abstracts, 1203 articles were excluded based on the selection criteria and
58 full text articles as well as two studies from updated search were assessed for eligibility $(n=60)$. In addition, the inter-rater reliability was measured by Cohen's kappa coefficient $(K=0.85 \pm 0.02)$ which has shown good agreement with the inclusion/exclusion criteria between the reviewers [28]. Following the review of the remaining articles and resolution of discrepancies by consensus among reviewers (ZB and BG), 11 studies were finally included in this systematic review.

\section{General characteristics of the study}

We analyzed 11 studies [2, 7, 17-25] with 94 hepatic GSD patients (72 Male, 22 Female). The detailed characteristics and outcomes of the included studies are

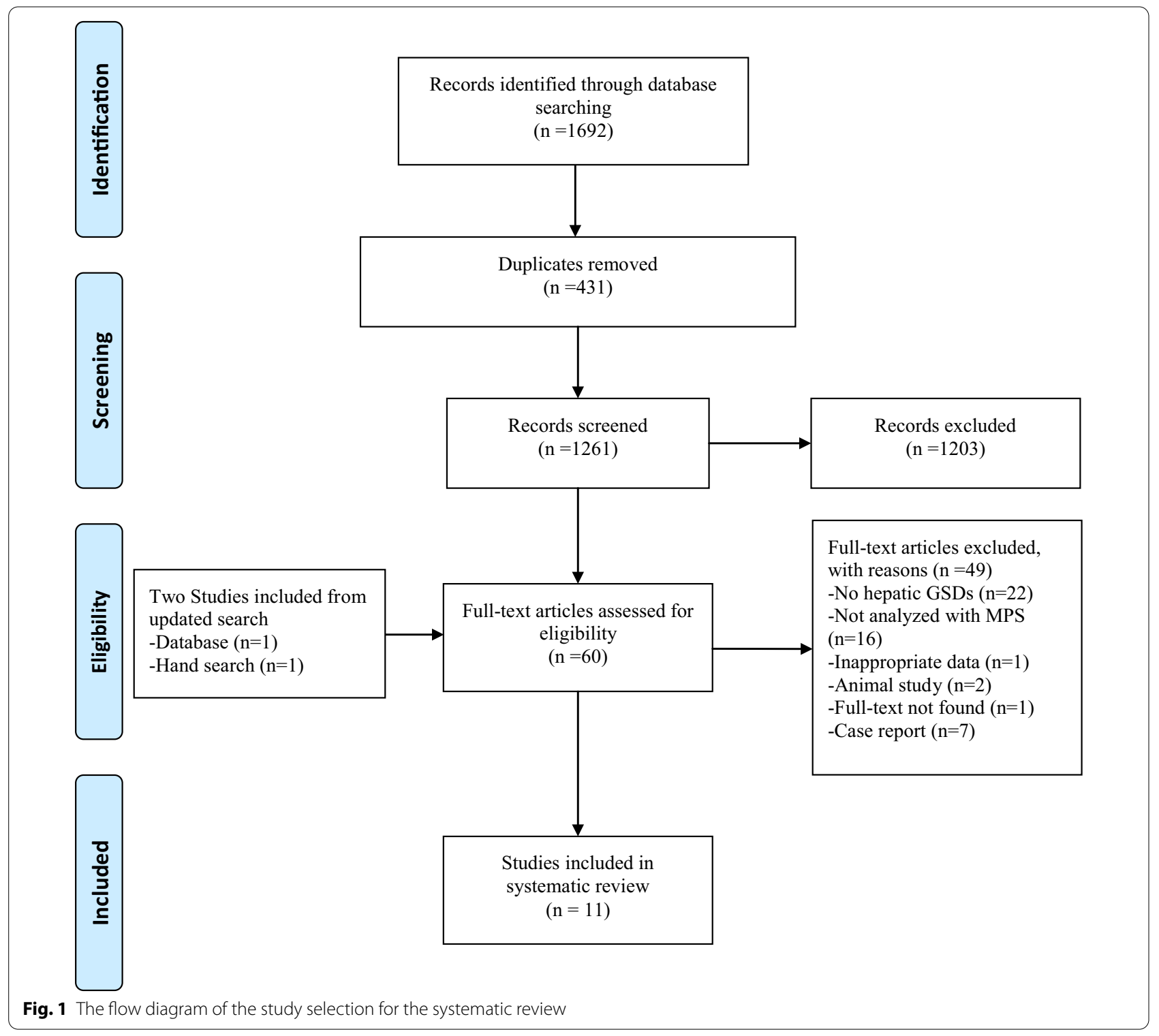


summarized in Table 1. All eleven studies analyzed in this article were published between 2013 and 2019, and contained 5 retrospective cohorts $[7,17,20,23,24], 4$ crosssectional $[2,18,19,21]$, and 2 case series [22, 25]. Five studies [17-19, 24, 25] included Asian participants, such as Chinese, Korean and Qatari, and the remaining 6 studies $[2,7,20-23]$ included Western participants, including 2 American, 1 Serbian, 1 Spanish, and 2 Canadian. Those 94 participants analyzed by MPS included hepatic GSD disease patients (2 GSD Ia, 7 GSD Ib, 16 GSD III, 7 GSD VI, 42 GSD IXa, 4 GSD IXb, 5 GSD IXc) and 7 non-GSD patients, and 4 patients with no exact diagnosis (Fig. 2a). GSD type IV participants with neuromuscular involvement and without liver involvement were excluded. The mean age of the disease onset was 2.1 years (Ranges: 1-35 months), and at the time of the molecular diagnostic test it was 5.8 years (Ranges: 10 months to 41 years).

The families of 69 patients, i.e. 96\% (69/72) were nonconsanguineous. Enzyme activity was analyzed in 46\% (43/94) of patients. There was no report of enzyme assay in $28.7 \%(27 / 94)$ of the patients. All results described above are presented in Table 1.

\section{Synthesis of results}

ES was used in 54.5\% (6/11) of articles [17-23], TGS in $36.4 \%(4 / 11)[2,20,24,25]$, and a combination of TGS and ES in 9.1\% (1/11), as shown in Table 1 [7]. Seven studies [2, 18-22, 24] used Illumina Hiseq 2000; one study [17] used Illumina Hiseq 2500 and in two studies $[7,23]$ Miseq Illumina was the sequencing platform. Two out of 11 studies analyzed single-end reads with an average depth of $>600 \times[2,21]$, while all other studies analyzed paired-end reads with an average depth of $>100 \times$. As presented in Table 1, sequence analysis of proband-parent trios was performed in the majority of patients $58.5 \%$ (55/94); as also a duo of proband and sibling was reported in $2.1 \%(2 / 94)$ of patients [7, 17, 20, 22, 25]. 39.4\% (37/94) of investigations were reported analyzing the proband alone (Fig. 2b).

Eighty-four percent (79/94) of patients were analyzed with TGS and sixteen percent (15/94) using ES methods. Our results showed that the overall diagnostic rate of ES, and TGS was 93\% (14/15), 79.7\% (63/79) for the detection of mutations in hepatic GSDs patients, respectively. Our findings demonstrated that by using ES methods, $100 \%$ of five patients with complex features were identified with a mutation in a GSD disease-associated gene although those patients were initially diagnosed as suffering from neurodevelopmental or other metabolic disorders. By application of the ES method, common mutations in the 5 patients were diagnosed with the non-GSD-associated disease, which was incorrectly diagnosed as hepatic GSDs $[7,23]$. The detected genes were LIPA and SBDS, CPT II,
$A N O 5$, and NKX2-5 which are the genes responsible for cholesteryl-ester storage disease, Schwachman-Diamond syndrome, carnitine palmitoyl transferase II deficiency, muscle disease (Limb-girdle muscular dystrophy type $2 \mathrm{~L}$ and Miyoshi muscular dystrophy 3 ) and congenital heart disease, respectively.

Three studies [20, 24, 25], analyzing hepatic GSD patients with the TGS method, found that among 41 patients, 30 could be detected with mutations in the average depth of sequence $\geq 400 \times$ and average diagnostic yield of approximately $73.1 \%(30 / 41)$. It is noted that by increasing the sequencing average depth to $>1000 \times$, the diagnostic yield of TGS could be enhanced to $86.8 \%$ (33/38). Also, the diagnostic yield of TGS by performing a trio-proband test could be increased from 64.5 to $79.7 \%$. In $20.3 \%$ of patients (16/79) analyzed by the TGS method, no mutations were diagnosed.

Finally, forty-six percent of patients (43/94) had undergone liver biopsy before the molecular genetic test which was performed in forty-four percent (35/79) of TGS patients and fifty-three (8/15) of ES patients. There was no liver biopsy for $25.4 \%$ (24/94) of the patients. In $28.7 \%$ (27/94) of the patients, nothing was reported about the performance of liver biopsy. However, the features of liver histopathology in $41.8 \%$ (18/43) of patients were not consistent with the molecular genetic investigations. In 10 patients, features of liver histopathology were suggestive of GSD-III, but molecular genetic investigations confirmed the diagnosis of GSD-IXa in 4, diagnosis of GSD-IXc in 2, and diagnosis of GSD-VI in 4 patients. In 2 patients, features of liver histopathology were suggestive of GSD-IV, but molecular genetic results confirmed the diagnosis of GSD-IXa in 1 patient and diagnosis of GSD-VI in another patient. In addition, features of liver histopathology were suggestive of GSD-Ia in one, GSD-0 in one patient, and GSD-VI in one patient, but molecular genetic investigations confirmed the diagnosis of GSD-Ib in one, GSD-IXc in one, and GSD-IXa in one patient, respectively. In 3 patients, features of liver histopathology were suggestive of liver disorder and hepatic GSD without exact sub-typing, but molecular genetic investigations confirmed the diagnosis of GSD-IXa in one, cholesteryl-ester storage disease in one, and Schwachman-Diamond syndrome in one patient, respectively.

\section{Discussion}

In this systematic review of 11 studies, our goal was to determine the diagnostic value of MPS as the first method of choice in GSDs with liver involvement. According to the results, most patients with hepatic GSDs are not provided with a specific molecular diagnostic test as the first approach of choice. The increased mean age of patients in 


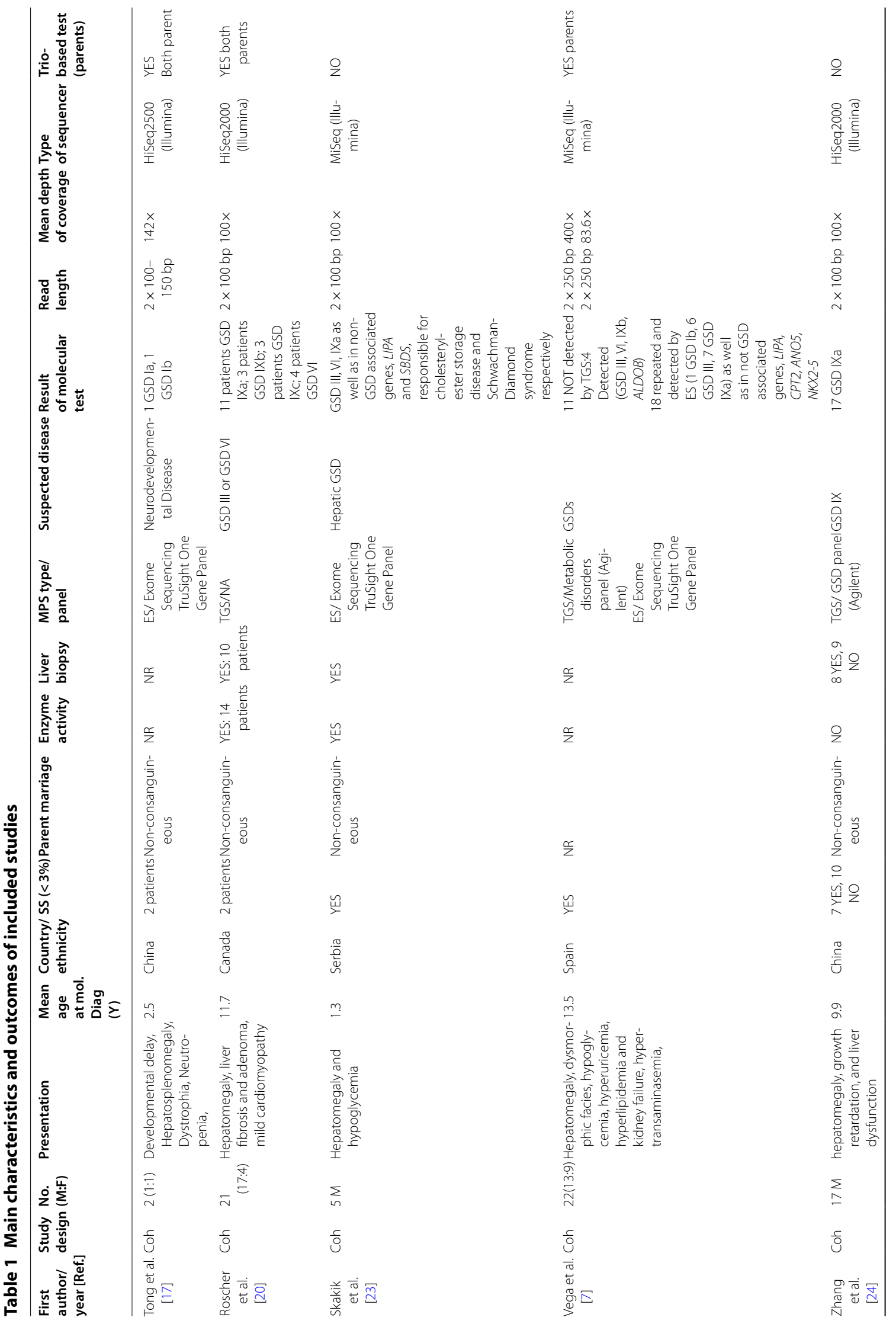




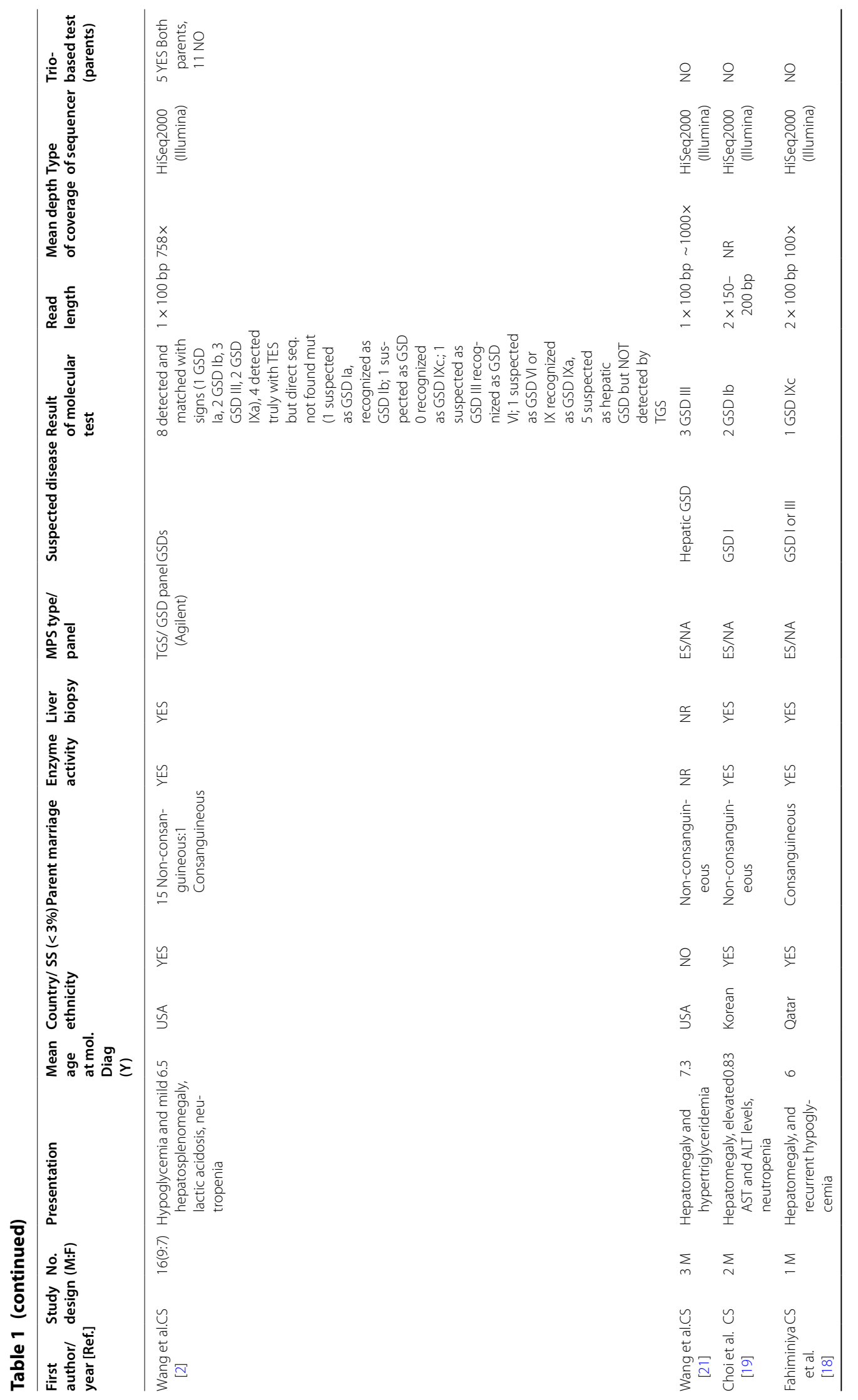




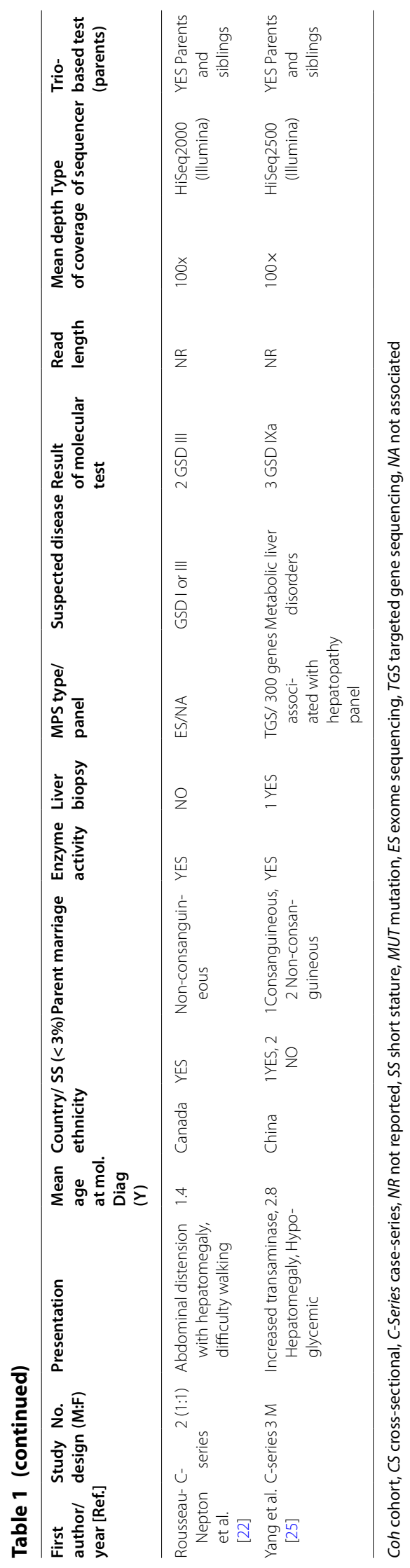



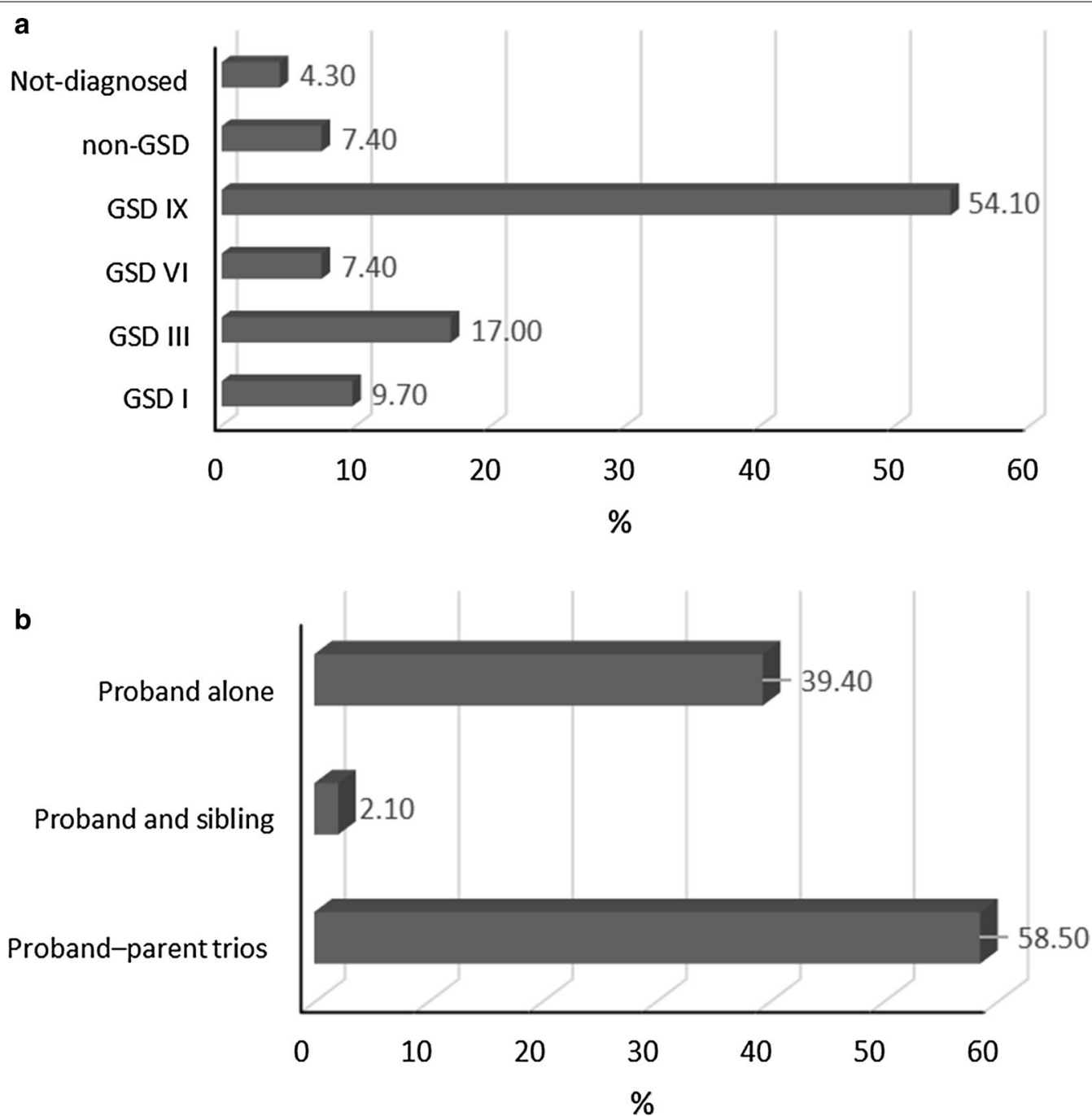

Fig. 2 The percentage of patients was diagnosed with MPS method (a) and the percentage of Trio-based test was performed (b)

disease onset in comparison with the time of a molecular performance from 2.1 to 5.8 emphasizes that this method has not been used as the first-line diagnostic method.

The standard approach for the diagnosis of hepatic GSDs is to identify specific phenotypic and clinical presentation of the disease, with consideration of liver biopsy findings, as well as of the tissue enzyme assay [1, 2, 29]. The main features of hepatic GSD are hepatomegaly and hypoglycemia, which delay the diagnosis and lead to chronic liver disease, e.g. fibrosis and cirrhosis [29]. It is worth noting that delayed diagnosis could have a harmful outcome in patients and their families leading to delayed treatment, and delayed recognition of the risk of recurrence in later pregnancies to protect younger siblings of the proband. In addition, other diagnostic methods can be time-consuming, invasive, and costly. Based on our results, the feature of liver histopathology may be a powerful and effective method for monitoring long-term liver complications, but not for confirming the diagnosis and accurate sub-typing. Therefore, the development of molecular method based on MPS may be valuable for an accurate diagnosis [30,31]. It should be noted that the clinical availability of MPS methods is limited and is only implemented in developed countries [32]. This is because of the high cost of the procedure.

According to our results, $54.5 \%$ of the studies opted to look for mutations by exome sequencing (ES), which is considered as an effective method with $100 \%$ sensitivity for determining unknown coding mutations. All studies surveyed were carried out with the same mean coverage of the ES method (100-150x), which suggests an appropriate mean coverage for ES. Furthermore, with the discovery of the causative gene, ES is also an effective diagnostic tool whenever no diagnosis could 
be made or an incorrect diagnosis has been reported based on clinical manifestations [33]. For example, our results demonstrate that ES could identify $73.3 \%$ of the mutations in the disease-associated gene although the patient was suspected to have other types of GSDs based on the preliminary clinical diagnosis. Diagnosing the correct type of hepatic GSDs not only influences the prognosis and care but also allows suitable genetic counseling to the family [34]. Our results demonstrate the role of ES in the detection of novel variants of complex features of hepatic GSDs, and advocate a role for trio-ES in detection of unknown variants. On the other hand, the use of ES panels increases the rate of accurate diagnosis [35, 36]. The ES method turned out to be the test with the highest diagnostic yield, especially when accompanied by a trio-based test, with $93 \%$ sensitivity, as reported earlier [21]. Likewise, performing ES methods using an Illumina Clinical-Exome Sequencing TruSight One Gene Panel identified the patients with non-GSD disease. According to studies reported these genes (LIPA, SBDS, CPT II, ANO5, NKX2) which had mutations, screened by ES methods. Those genes are responsible for cholesteryl-ester storage disease, Schwachman-Diamond syndrome, carnitine palmitoyl transferase II deficiency, muscle disease (Limb-girdle muscular dystrophy type $2 \mathrm{~L}$ and Miyoshi muscular dystrophy 3 ), and congenital heart disease respectively. These genes have no demonstrated role in GSDs, and they are manifested with overlapping phenotypic characteristics, such as hypoglycemia, seizures, hepatomegaly, cardiomyopathy, and arrhythmia.

The other method of MPS, TGS also detected pathogenic variants but its sensitivity was very different. It is noted that TGS and ES are used to perform targeted exome sequencing of metabolic disorders, including GSDs-associated genes with/or without genes related to its pathological phenotypes and all known disease-associated genes, rather than the entire exome, respectively [37-39]. Our results show that the diagnostic rate of TGS is $79.7 \%$, though the rate may be affected by the type of panel selected, depth of sequencing coverage, and whether other family members are analyzed. The selection of the appropriate panels covering a wide range of similar GSD-genes is very important which shows different diagnostic yields for the TGS method in different reports [2, 7, 20, 24, 25]. It is probably explained by the high resemblance of GSD to other non-GSDs disorders with phenotype overlapping. In the undiagnosed cases by TGS, the mutations probably existed in coding regions that were not adequately covered, or they could be located within deep intronic regions that were not covered by TGS or might be within non-GSD-associated genes. Also, we found that increasing the depth of the sequence coverage enhanced the diagnostic yield with the TGS method. Previous investigations have shown that diagnostic yield increases by performing capture-based enrichment, followed by deep sequencing (1000x) [2]. Capture/MPS allows detection of a wide spectrum of mutations [21]. This technology helps to detect all types of mutations such as single nucleotide substitutions, small insertion/deletions as well as exonic copy number variation, and large genomic rearrangements [38]. Our results also showed that analysis of trios performed in $60.6 \%$ of the patients, significantly enhanced the diagnostic yields, compared with proband-only testing, due to the heterogeneous genetic basis of hepatic GSDs. Consequently, the TGS method might be suitable for first-line molecular study of hepatic GSDs, and it is recommended to be performed only when presentations of disease are very clear, using a wide range of disease panels.

Therefore, the best diagnostic strategy to identify hepatic GSDs can be starting with a TGS method, as a more cost-effective method than the ES, but with the high coverage and a wide range of the panel. If there is no definite result, then analysis with a more comprehensive method, such as an ES, should be performed [40]. ES should particularly be the diagnostic tool of choice when an accurate diagnosis of more complex cases is necessary. To note, ES is known to bias coverages based on capture reagent and large rearrangements, which are extremely difficult to detect. Therefore, a recent publication reported that chromosomal microarray (CMA) testing followed by ES could improve the yield of genetic diagnosis [41, 42]. Analytical workflows for the diagnosis of GSD diseases are not fully standardized, so we recommended it, as shown in Fig. 3.

There were some limitations to the present study. First, the number of studies was very small, so the small sample size of patients restricted confidence in our analysis. Second, all included studies were observational and retrospectively selected cohort studies with the small number of patients, which could be resulted in the selection bias of patients. Finally, we only included English studies that result in missing the studies with other languages and not indexed in the databases.

\section{Conclusions}

The correct characterization of clinical, biochemical, and pathological patterns of patients is important in order to interpret the genetic results. However, the MPS method could be a step forward in terms of enabling correct diagnosis of hepatic GSDs. All the patients mentioned in the analyzed reports, were offered genetic and metabolic assessments, including liver biopsy, enzyme assay, and single-gene sequencing prior to MPS. The present work demonstrates that, despite 


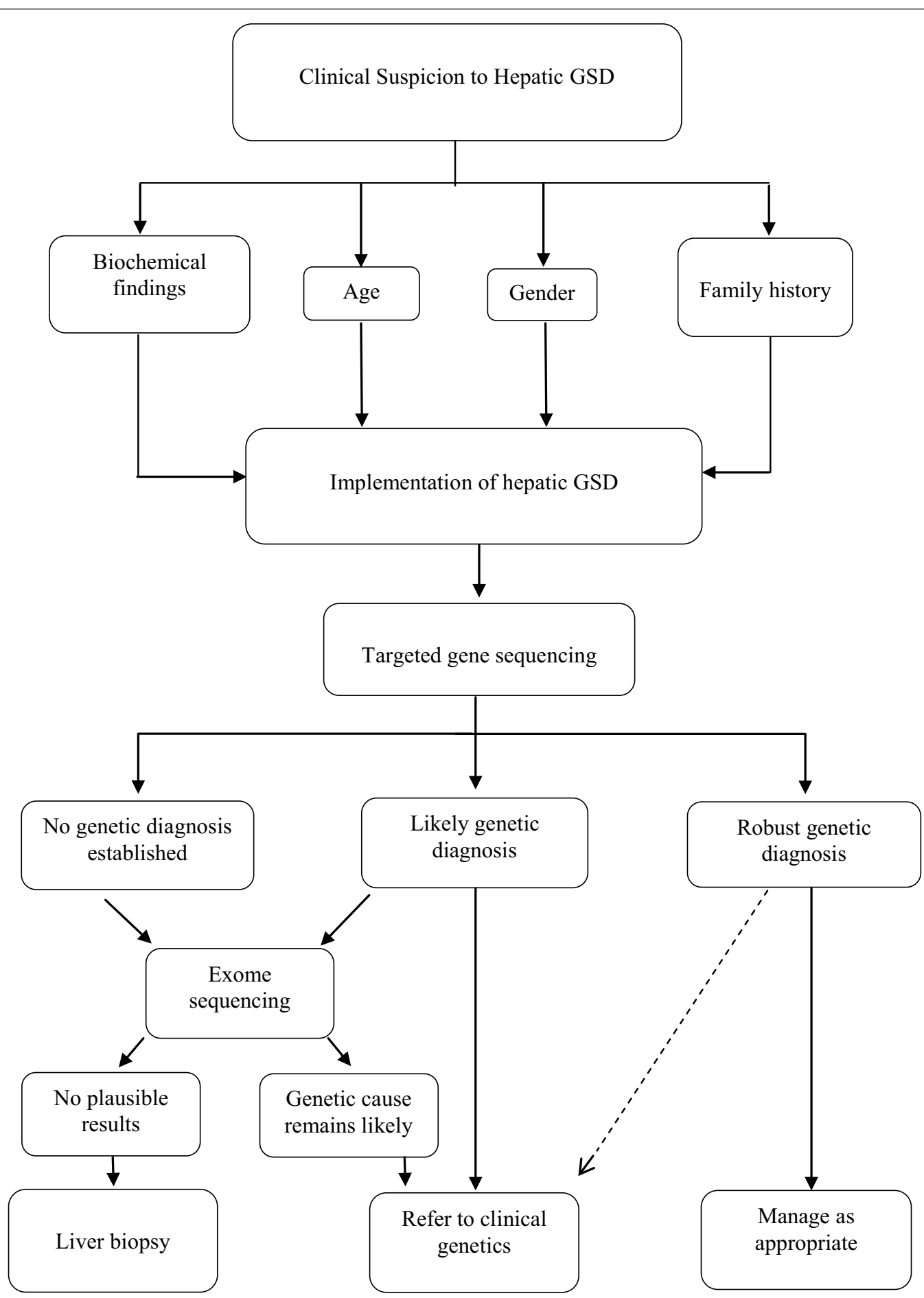

Fig. 3 Integration of clinical and laboratory workflows to optimize the hepatic glycogen storage disease diagnosis

its cost, the time effectiveness and accuracy of MPS in the diagnosis of hepatic GSDs could avoid incorrect and/or delayed treatment of patients. We propose that TGS may be considered as the first-line method of choice for diagnosis of hepatic GSDs with a wide range of panel, as it allows the detection of pathogenic variants in GSD-associated vs. non-GSD-associated genes 
with overlapping symptoms in hepatic manifestations. It must be emphasized that with the extended use of TGS/ES strategies in finding the causes of liver disease, the so-called milder or adult forms of inborn errors can be accurately detected.

\section{Supplementary information}

Supplementary information accompanies this paper at https://doi. org/10.1186/s13023-020-01573-8.

Additional file1: Supplementary Table 1. PRISMA 2009 Checklist. Sup plementary Table 2. Search strategy for MEDLINE/PubMed, EMBASE, Cochrane Library, Scopus and Web of Science Core Collection databases. Supplementary Table 3. Quality assessment scores according to the NHLBI Quality Assessment Tool for Observational Cohort, Cross-Sectional and Case series Studies for each reviewer.

\section{Abbreviations}

GSD: Glycogen storage diseases; MPS: Massively parallel sequencing; ES: Exome sequencing; TGS:Targeted gene sequencing; CMA: Chromosomal microarray.

\section{Acknowledgements}

We are immensely grateful to Mahsa Marzban, University of British Columbia for her invaluable assistance in editing this manuscript.

\section{Authors' contributions}

ZB: served as primary investigator for the study, helped to design the study, directed data collection, analyzed data and interpretation, created first draft of manuscript, and edited the manuscript. SK: Collected data. BG: Senior author, created the project, coordinated data collection, critically revised the work, and edited the manuscript. All authors read and approved the final manuscript.

\section{Funding}

This study was financially supported by Transplant Research Center, affiliated with Shiraz University of Medical Sciences, Shiraz, Iran (Grant No.20458).

\section{Availability of data and materials}

The datasets used and/or analyzed during the current study are available from the corresponding author on reasonable request.

\section{Ethics approval and consent to participate}

Not applicable.

\section{Consent for publication}

Not applicable.

\section{Competing interests}

The authors declare that they have no competing interests.

\section{Research involving human participants and/or animals}

This article is a systematic review, so it does not contain any studies with human participants or animals performed by any of the authors.

\section{Informed consent}

For this type of study, formal consent is not required.

\section{Author details}

${ }^{1}$ Transplant Research Center, Shiraz University of Medical Sciences, Shiraz, Iran. ${ }^{2}$ Department of Pathology, Shiraz University of Medical Sciences, Zand St., Shiraz, Iran. ${ }^{3}$ Shiraz Medical School Library, Shiraz University of Medical Sciences, Shiraz, Iran.
Received: 1 February 2020 Accepted: 5 October 2020

Published online: 14 October 2020

\section{References}

1. Beyzaei Z, Geramizadeh B. Molecular diagnosis of glycogen storage disease type I: a review. EXCLI J. 2019;18:30-46.

2. Wang J, Cui H, Lee NC, Hwu WL, Chien YH, Craigen WJ, Zhang WW. Clinical application of massively parallel sequencing in the molecular diagnosis of glycogen storage diseases of genetically heterogeneous origin. Genet Med. 2013;15(2):106-14.

3. Bhattacharya K. Investigation and management of the hepatic glycogen storage diseases. Transl Pediatr. 2015;4(3):240-8.

4. Boycott MRV, Bulman DE, MacKenzie AE. Rare-disease genetics in the era of next-generation sequencing: discovery to translation. Nat Rev Genet. 2013;14(10):681-91.

5. McCandless SE, Brunger JW, Cassidy SB. The burden of genetic disease on inpatient care in a children's hospital. Am J Hum Genet. 2004;74:121-7.

6. Burda P, Hochuli M. Hepatic glycogen storage disorders: what have we learned in recent years? Curr Opin Clin Nutr Metab Care. 2015;18(4):15-421.

7. Vega CM, Navarrete R, Desviat LR, Merinero B, Rodríguez-Pombo P, Vitoria I, Ugarte M, Pérez-Cerdá C, Pérez B. Molecular diagnosis of glycogen storage disease and disorders with overlapping clinical symptoms by massive parallel sequencing. Genet Med. 2016;18(10):1037-43.

8. Shin YS. Glycogen storage disease: clinical, biochemical, and molecular heterogeneity. Semin Pediatr Neurol. 2006;13(2):115-20.

9. Neg SB, Turner EH, Robertson PD, et al. Targeted capture and massively parallel sequencing of twelve human exomes. Nature. 2009:461(7261):272-6.

10. Schwarze K, Buchanan J, Taylor JC, Wordsworth S. Are whole-exome and whole-genome sequencing approaches cost-effective? A systematic review of the literature. Genet Med. 2018;20(10):1122-30.

11. Davies SC. Annual report of the chief medical officer 2016, generation genome. London: Department of Health; 2017.

12. Smith HS, Swint JM, Lalani SR, Yamal JM, de Oliveira Otto MC, Castellanos S, Taylor A, Lee BH, Russell HV. Clinical application of genome and exome sequencing as a diagnostic tool for pediatric patients: a scoping review of the literature. Genet Med. 2019;21(1):3-16.

13. Nicastro $E, D^{\prime}$ Antiga L. Next generation sequencing in pediatric hepatology and liver transplantation. Liver Transpl. 2018;24(2):282-93.

14. Black N. Why we need observational studies to evaluate the effectiveness of health care. BMJ. 1996;312:1215-8.

15. Mueller M, D'Addario M, Egger M, Cevallos M, Dekkers O, Mugglin C, Scott P. Methods to systematically review and meta-analyses observational studies: a systematic scoping review of recommendations. BMC Med Res Methodol. 2018:18(1):44.

16. Sims D, Sudbery I, llott NE, Heger A, Ponting CP. Sequencing depth and coverage: key considerations in genomic analyses. Nat Rev Genet. 2014;15(2):121-32.

17. Tong W, Wang $Y, L u Y, Y e T$, Song $C, X u Y$, Jin D. Whole-exome sequencing helps the diagnosis and treatment in children with neurodevelopmental delay accompanied unexplained dyspnea. Sci Rep. 2018;8(1):5214-23.

18. Fahiminiya S, Almuriekhi M, Nawaz Z, Staffa A, Lepage P, Ali R, Hashim L, Schwartzentruber J, Abu Khadija K, Zaineddin S, Gamal H, Majewski J, Ben-Omran T. Whole exome sequencing unravels disease-causing genes in consanguineous families in Qatar. Clin Genet. 2014;86(2):134-41.

19. Choi R, Park HD, Ko JM, Lee J, Lee DH, Hong SJ, Ki CS, Lee SY, Kim JW, Song J, Choe YH. Novel SLC37A4 mutations in Korean patients with glycogen storage disease Ib. Annals Lab Med. 2017;37(3):261-6.

20. Roscher A, Hewson S, Nagy L, Feigenbaum A, Kronick J, Raiman J, Schulze A, Siriwardena K, Mercimek-Mahmutoglu S. The natural history of glycogen storage disease types VI and IX: long-term outcome from the largest metabolic center in Canada. Mol Genet Metab. 2014;113:171-6.

21. Wang J, Yu H, Zhang VW, Tian X, Feng Y, Wang G, Wong LJ. Capture-based high-coverage NGS: a powerful tool to uncover a wide spectrum of mutation types. Genet Med. 2016;18(5):513-21. 
22. Rousseau-Nepton I, Okubo M, Grabs R, Boycott K, Friedman J, Michaud J, Rodd C. A founder AGL mutation causing glycogen storage disease type IIla in Inuit identified through whole-exome sequencing: a case series. CMAJ. 2015;187(2):68-73.

23. Skakic A, Djordjevic M, Sarajlija A, Klaassen K, Tosic N, Kecman B, Stojiljkovic M. Genetic characterization of GSD I in Serbian population revealed unexpectedly high incidence of GSD Ib and 3 novel SLC37A4 variants. Clin Genet. 2018;93(2):350-5.

24. Zhang J, Yuan Y, Ma M, Liu Y, Zhang W, Yao F, Qiu Z. Clinical and genetic characteristics of 17 Chinese patients with glycogen storage disease type IXa. Gene. 2017;627:149-56.

25. Yang F, Xu Y, Fang C, Tan L, Tan Q, Zhou Y. Clinical and genetic characteristics of three Chinese patients with glycogen storage disease type IXa . Pediatr Neonatol. 2019:60:463-6.

26. Moher D, Shamseer L, Clarke M, Ghersi D, Liberati A, Petticrew M, Shekelle $P$, Stewart LA. Preferred reporting items for systematic review and metaanalysis protocols (PRISMA-P) 2015 statement. Syst Rev. 4, 1.

27. National Heart, Lung, and Blood Institute (NHLBI). Quality Assessment Tool for Observational Cohort and Cross-Sectional Studies. Bethesda, MD: National Heart, Lung, and Blood Institute, 2014. https://www.nhlbi. nih.gov/healthpro/guidelines/in-develop/cardiovascular-risk-reduction/ tools/cohort.

28. McHugh ML. Interrater reliability: the kappa statistic. Biochem Med (Zagreb). 2012:22:276-82.

29. Hicks J, Wartchow E, Mierau G. Glycogen storage diseases: a brief review and update on clinical features, genetic abnormalities, pathologic features, and treatment. Ultrastruct Pathol. 2011;35:183-96.

30. Bentley DR. Accurate whole human genome sequencing using reversible terminator chemistry. Nature. 2008:456:53-9.

31. Wang DQ, Fiske LM, Carreras CT, Weinstein DA. Natural history of hepatocellular adenoma formation in glycogen storage disease type I. J Pediatr. 2011;159:442-6.

32. Wright $C F$, FitzPatrick DR, Firth HV. Pediatric genomics: diagnosing rare disease in children. Nat Rev Genet. 2018;19(5):253-68.

33. Moorthie S, Mattocks CJ, Wright CF. Review of massively parallel DNA sequencing technologies. HUGO J. 2011;5:1-12.
34. Chinault AC, Shaw CA, Brundage EK, Tang LY, Wong LJ. Application of dual genome oligonucleotide array-based comparative genomic hybridization to the molecular diagnosis of mitochondrial DNA deletion and depletion syndromes. Genet Med. 2009;11:518-26.

35. Lee H, Deignan JL, Dorrani N, Strom SP, Kantarci S, Quintero-Rivera F, Das K, Toy T, Harry B, Yourshaw M, Fox M, Fogel BL, Martinez-Agosto JA, Wong DA, Chang VY, Shieh PB, Palmer CG, Dipple KM, Grody WW, Vilain E, Nelson SF. Clinical exome sequencing for genetic identification of rare Mendelian disorders. JAMA. 2014;312(18):1880-7.

36. Yang Y, Muzny DM, Xia F, et al. Molecular findings among patients referred for clinical whole-exome sequencing. JAMA. 2014;312:1870-9.

37. Yang Y, Muzny DM, Reid JG, et al. Clinical whole-exome sequencing for the diagnosis of mendelian disorders. N Engl J Med. 2013;369:1502-11.

38. Yavarna T, Al-Dewik N, Al-Mureikhi M, et al. High diagnostic yield of clinical exome sequencing in Middle Eastern patients with Mendelian disorders. Hum Genet. 2015:134:967-80.

39. Valencia CA, Husami A, Holle J, et al. Clinical impact and cost-effectiveness of whole exome sequencing as a diagnostic tool: a pediatric center's experience. Front Pediatr. 2015;3:67.

40. Kodabuckusa SS, Quinlan-Jonesb E, McMullanc DJ, et al. Exome Sequencing for Prenatal Detection of Genetic Abnormalities in Fetal Ultrasound Anomalies: An Economic Evaluation. Fetal Diagn Ther. 2020. https://doi. org/10.1159/000504976.

41. Srivastava S, Love-Nichols JA, Dies KA, et al. Meta-analysis and multidisciplinary consensus statement: exome sequencing is a first-tier clinical diagnostic test for individuals with neurodevelopmental disorders. Genet Med. 2019:21(11):2413-21.

42. Baldridge D, Heeley J, Vineyard $M$, et al. The Exome clinic and the role of medical genetics expertise in the interpretation of exome sequencing results. Genet Med. 2017;19:1040-8.

\section{Publisher's Note}

Springer Nature remains neutral with regard to jurisdictional claims in published maps and institutional affiliations.
Ready to submit your research? Choose BMC and benefit from:

- fast, convenient online submission

- thorough peer review by experienced researchers in your field

- rapid publication on acceptance

- support for research data, including large and complex data types

- gold Open Access which fosters wider collaboration and increased citations

- maximum visibility for your research: over $100 \mathrm{M}$ website views per year

At BMC, research is always in progress.

Learn more biomedcentral.com/submissions 